\title{
Analytical Modeling of Residual Stress in Selective Laser Melting Considering Volume Conservation in Plastic Deformation
}

\author{
Elham Mirkoohi ${ }^{1,}$, Dongsheng $\mathrm{Li}^{2}{ }$, Hamid Garmestani ${ }^{3}$, and Steven Y. Liang ${ }^{1}$ \\ ${ }^{1}$ Woodruff School of Mechanical Engineering, Georgia Institute of Technology, Atlanta, GA 30332, USA; \\ steven.liang@me.gatech.edu and emirkoohi3@gatech.edu \\ ${ }^{2}$ Advanced Manufacturing LLC, 222 Pitkin St, East Hartford, CT 06108; dongshengli@gmail.com \\ ${ }^{3}$ School of Material Science and Engineering, Georgia Institute of Technology, Atlanta, GA 30332, USA; \\ h.garmestani@gmail.com \\ * Correspondence: emirkoohi3@gatech.edu
}

\begin{abstract}
Residual stress (RS) is the most challenging problem in metal additive manufacturing (AM) since the build-up of high tensile RS may influence the fatigue life, corrosion resistance, crack initiation, and failure of the additively manufactured components. While tensile RS is inherent in all the AM processes, fast and accurate prediction of stress state within the part is extremely valuable and would result in optimization of the process parameters in achieving a desired RS and control of the build process. This paper proposes a physics-based analytical model to rapidly and accurately predict the RS within the additively manufactured part. In this model, a transient moving point heat source (HS) is utilized to determine the temperature field. Due to the high temperature gradient within the proximity of the melt pool area, material experience high thermal stress. Thermal stress is calculated by combining three sources of stresses known as stresses due to the body forces, normal tension, and hydrostatic stress in a homogeneous semi-infinite medium. The thermal stress determines the RS state within the part. Consequently, by taking the thermal stress history as an input, both the in-plane and out of plane RS distributions are found from incremental plasticity and kinematic hardening behavior of the metal by considering volume conservation in plastic deformation in coupling with the equilibrium and compatibility conditions. In this modeling, material properties are temperature-sensitive since the steep temperature gradient varies the properties significantly. Moreover, the energy needed for the solid-state phase transition is reflected by modifying the specific heat employing the latent heat of fusion. Furthermore, the multi-layer and multi-scan aspects of metal AM are considered by including the temperature history from previous layers and scans. Results from the analytical RS model presented excellent agreement with XRD measurements employed to determine the RS in the Ti-6Al-4V specimens.
\end{abstract}

Keywords: Selective Laser Melting; residual stress; direct metal deposition; thermomechanical analytical modeling; Ti-6Al-4V

\section{Introduction}

Over the last decade, metal Additive Manufacturing (AM) has developed rapidly to become a revolutionize technology for the production of various components for several industries such as 
biomedical, aerospace, automotive, and marine as stated by Herderick [1]. Namatollahi et al. [2] stated that "AM is the process of joining metallic powders layer by layer to produce complex threedimensional parts". AM has several advantageous over conventional manufacturing including lower density, reduction in scrap rate, complex nearly net-shape parts, elimination of multi-step manufacturing, and many more as expressed by Camacho et al. [3]. In contrast, there are still some limitations that hinder the applicability of AM such as steep temperature gradient, high thermal stress, and tensile residual stress (RS) as reported by Ngo et al. [4], anisotropy and heterogeneity in microstructure as explained in the work of Ji et al. [5] and mechanical properties as explained in the work of Tabei et.al [6].

Bartlett et al. [7] described that steep temperature gradient induced by high laser power and low conduction may cause high thermal stress in the component. Since the yield strength of the material depends on temperature, and at elevated temperatures around the melting point, materials are usually soft, they can easily undergo plastic deformation. Consequently, due to the repeated heating and cooling, material experience a high magnitude of RS. Residual stress is inherent in all the manufactured components, and high RS is reported by many researchers. Roehling et al. [8] reported high tensile RS in parts built via powder bed fusion (LPBF) systems. Wang et al. [9] measured the RS using neutron diffraction, they observed high tensile RS in IN625 parts. An et al. [10] reported high RS in curved thin-walled structure manufactured via LPBF. Denlinger and Heigel [11] simulated the RS during the additively manufactured Ti-6Al-4V samples, they stated that the predicted RS was considerably high. Zhao et al. [12] predicted high RS in titanium alloys build via direct metal laser sintering (DMLS) using FEM. Romano et al. [13] reported that RS has a crucial impact on fatigue life of the component, corrosion resistance, crack initiation and growth, and also microstructure and mechanical properties of the materials.

There is a considerable amount of research on literature explaining different methods for the prediction of RS including experimentation, numerical modeling, and analytical modeling.

Experimental procedures to measure the RS in the components could be categorized into destructive and non-destructive methods. The non-destructive methods can be classified into X-ray diffraction- which is cable of near surface RS measurements- and neutron diffraction- which is capable of volumetric measurements. Other non-destructive methods include ultra-sonics as explained by Noronha and Wert [14], electrical resistivity as explained by Chung [15] magnetic behavior as explained by Krause et al. [16] and piezo-spectroscopy in thin films as explained by Ager III and Drory [17]; these are material and geometry specific. Destructive methods such as hole drilling, sectioning, crack compliance, digital image correlation, and electronic speckle pattern interferometry (ESPI) essentially create a free surface in the part and correlate resultant deformation to RS as stated by Prime [18].

Numerical modeling is another approach for the forecast of RS. Aggarangsi and Beuth [19] used a finite element method (FEM) to simulate the RS. In their modeling, they defined a temperature gradient from the melt pool and correlated it to the maximum RS. They have shown that localized preheating could reduce the maximum RS in the additively manufactured 304 stainless steel. Panda and Sahoo [20] used FEM to predict the RS in the direct metal deposition (DMD) of AlSi10Mg. They have predicted temperature distribution using transient temperature and coupled the results to a structural model to predict the RS. In their modeling, the thermal and mechanical material properties are considered constant. Also, the effects of scan strategies are not considered in this modeling. Chen et al. [21] used FEM to simulate the RS in additively manufactured parts using inherent strain method. They have utilized the temperature results obtained from thermocouples to calibrate the process parameters. Then, they have used Goldak's HS model to obtain the thermal gradients and the inherent strains. Ganeriwala et al. [22] also used FEM to simulate the stress state in laser powder bed fusion of Ti-6Al-4V. They have used lumping approach to speed up the computations. They indicated that the stress is higher near the boundaries for the island scan strategies. Ding and Shin [23] proposed a 3D thermo-elastoplastic finite element model to simulated the RS. They have validated the results using neutron diffraction strain scanner.

Physics-based closed-form analytical solutions are the other approach for the prediction of RS. Prediction of RS should be started by modeling of the temperature field induced by laser in AM. 
There are various analytical methods to model the temperature field in metal AM. Mirkoohi et al. [24] introduced five distinct HS models to analytically simulate the three-dimensional temperature profile. They have investigated the viability of all the models under various process conditions. They have considered the material properties temperature-dependent; phase change is also considered through modification of specific heat. Furthermore, the effect of scan strategy is also considered in their modeling. Mirkoohi et al. [25] have proposed a model to analytically simulate the temperature field considering the effect of layer thickness. Fergani et al. [26] presented a model for the prediction of stress. In their modeling, properties are considered constant. Also, the effect of scan strategy is not considered in their modeling. Moreover, the stress in the build direction is considered to be elastic.

Experimentation is a different approach which facilitates the perception of RSin metal AM. But, measuring the stress state of the entire part is challenging, time-consuming, and expensive. Physicsbased numerical models are an assuring approach for the calculation of RS when the results are compared to experiments. However, the simulation of the entire process considering the multiphysics aspects of metal AM cannot be achieved in a reasonable amount of time. On the other hand, physics-based closed-form analytical solutions validated by physical experiments enable prediction of the stress state within the additively manufactured part much faster than FEM and experimentation. Furthermore, the multi-physics aspect of metal AM such as multi-layer and multiscan aspect of AM- which is challenging to consider by any other methods- can be considered in the process modeling of AM using closed-form analytical solutions. This accurate and reliable model can be adopted to optimize the AM parameters and guide the build process in achieving the desired part quality. Consequently, having one model to accurately and rapidly predict the stress state within the part is extremely valuable.

The physics governing the AM is considerably complicated, thus, capturing the entire physical phenomena involved in this process currently is not possible. In this work, a physics-based closedform analytical model is proposed to predict the RS within the additively manufactured part. Herein, the proposed thermomechanical model is capable of the prediction of RS accurately in a few seconds. Prediction of RS cannot be achieved within this amount of time by any numerical methods or experimentation, this shows the computational efficiency of the proposed model. In this work, first, a transient moving point HS is employed to analytically simulate the in-process temperature field within the components. Second, temperature gradient along the scan direction ( $x$-axis) and build direction (z-axis) is calculated from temperature field. Third, thermal stress is obtained by combining three different sources of stress known as stresses due to body forces, normal tension, and hydrostatic stress. Last, the RS distributions along the build direction and also along the scan direction are found from incremental plasticity and kinematic hardening behavior of the metal based upon the premises of plane strain condition in the build of isotropic and homogeneous properties. Moreover, the RS is calculated according to the conservation of volume in plastic deformation, in coupling with the equilibrium and compatibility conditions. In this modeling, the thermal and mechanical material properties are temperature-sensitive, since the steep temperature gradient causes the material properties to vary substantially. Moreover, due to the cyclic melting and solidification, the build part experiences phase change. The heat capacity is modified to consider the effect of latent heat of melting during this process. Furthermore, the impacts of multi-layer and multi-scan aspects of metal AM is analyzed by including the effect of temperature history on former layers and scans.

X-ray diffraction experimental measurements are conducted to validate the proposed analytical model. The Ti-6Al-4V samples are built using direct metal deposition (DMD) process under different process condition. Good agreement is obtained between measured and predicted RS.

\section{Process Modeling}

A fully coupled analytical thermomechanical model is proposed to predict the RS within the additively manufactured parts with less than 46 seconds with a four-core processor laptop. The high computational efficiency of the proposed model makes the optimization of the process parameters and control of the process in achieving a high-performance part possible. It also enables the inverse analysis of AM process to design the process parameters to reduce the RS or change the stress state 
of the part from tensile to compressive. Changing the stress state of the part from tensile to compressive increases the fatigue life of the components and eliminates the crack initiation and growth and as a result, eliminates part failure.

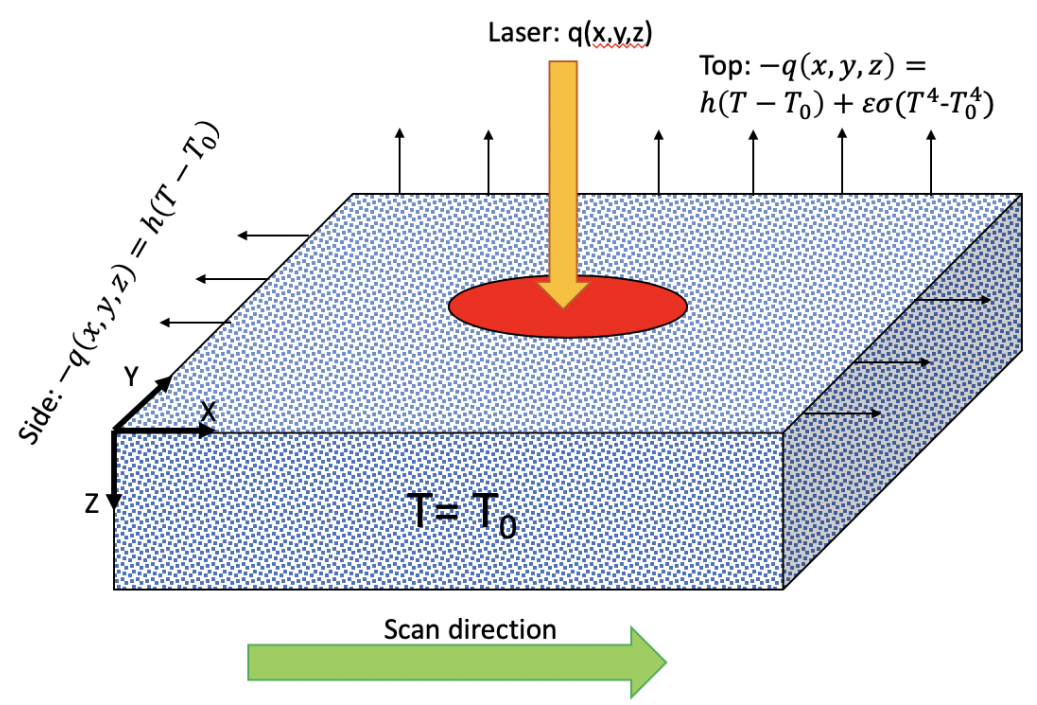

Figure 1. Heat transfer model of AM process.

Figure 1 illustrates the moving heat source $\mathrm{q}(\mathrm{x}, \mathrm{y}, \mathrm{z})$, which simulates the presence of the laser. As the laser moves along the surface according to the set scan strategy, it deposits the required energy for the melting the metallic powders. In this modeling, the effects of the multi-scan and multi-layer aspects of AM are considered by defining the hatching space and layer thickness. The hatching space and layer thickness are $103 \mu \mathrm{m}$ and $250 \mu \mathrm{m}$, respectively, and the scan strategy is bi-directional as shown in Figure 2.

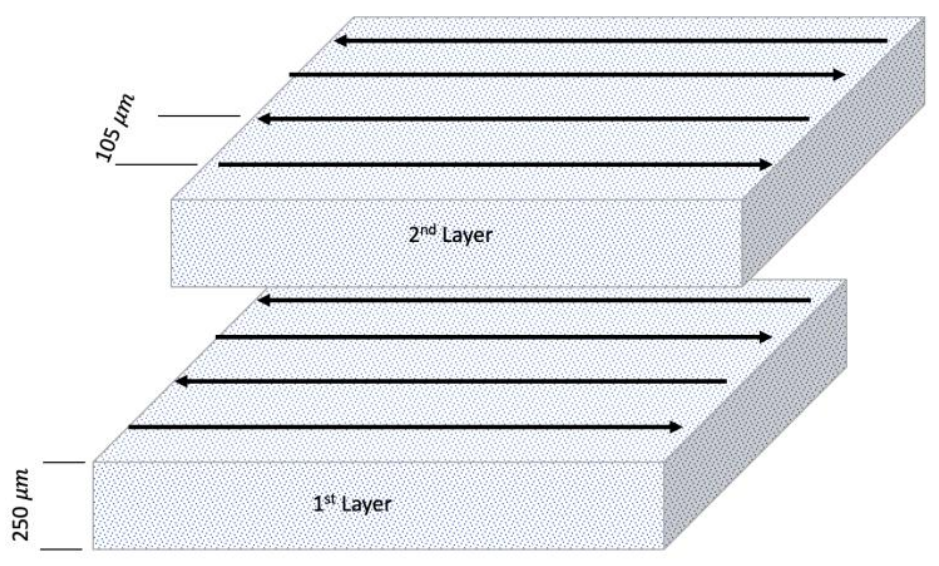

Figure 2. Illustration of layer height, hatch spacing, and scan strategy.

The steep temperature gradient considerably varies the material properties within the medium. Therefore, the properties of Ti-6Al-4V are temperature-sensitive. Moreover, material experience repeated melting and solidification during this process. The heat capacity is modified to consider the impact of latent heat of melting. Furthermore, many layers should be deposited and joined to produce a part. Therefore, the multi-layer features of metal AM, as well as layer width, are included by utilizing the temperature history from the former layers. Finally, the effect of multi-scan and hatching space is also considered by assessing the thermal history from the previous scans. Sections 2.1 and 2.2 represent the thermal and mechanical model used for the RS modeling. 


\subsection{Thermal Analysis}

Carslaw and Jaeger [27] for the first time introduced the transient temperature solution for the case of moving heat source by solving the differential equation of heat conduction;

$$
T=\frac{P \eta}{8 \rho C_{p}^{m}(\pi D t)^{3 / 2}} \int_{0}^{t} \frac{\exp \left(-\frac{\left(\left(x-x^{\prime}\right)-V\left(t-t^{\prime}\right)\right)^{2}+\left(y-y^{\prime}\right)^{2}+\left(z-z^{\prime}\right)^{2}}{4 D\left(t-t^{\prime}\right)}\right)}{\left(t-t^{\prime}\right)^{3 / 2}} d t^{\prime}+T_{0}
$$

Equation 1 provides the solution to analytically simulate the temperature distribution at position $(x, y, z)$ at time $t$ due to an instantaneous unit heat source applied at position $\left(x^{\prime}, y^{\prime}, z^{\prime}\right)$ at time $t^{\prime}$. The steady state moving heat source can be derived with infinite $t$. It can be expressed as

$$
T=\frac{P \eta}{4 \pi k R} \exp \frac{-V(R-x)}{2 D}+T_{0}
$$

where $P$ represents the laser power, $\eta$ represents the absorption coefficient, $\rho$ is density, $C_{p}^{m}$ is the modified heat capacity to consider the latent heat of fusion for solid-state phase change as explained in the work of Mirkoohi et al. [24]. $V$ is scan speed, $D$ is thermal diffusivity, and $T_{0}$ is the initial temperature.

\subsection{Mechanical Analysis}

Non-uniform heating induced by fast irradiation of the laser and low conduction, re-melting and re-solidification, and different thermal expansion coefficient induced by steep temperature gradient throughout the part are the main sources of thermal stress.

Considering the plain strain condition $\left(\varepsilon_{y y}=0\right)$, the normal strain along the scan direction can be obtained as;

$$
\varepsilon_{x x}=\frac{1}{E(T)}\left(1-v(T)^{2}\right) \sigma_{x x}+\alpha T(1+v(T))
$$

where the elastic modulus $\mathrm{E}(\mathrm{T})$, Poisson's ratio $v(T)$, and coefficient of thermal expansion $\alpha(T)$ are temperature dependent. $\sigma_{x x}$ is the normal stress along the scan direction. The acquired temperature field could then be used to calculate the thermal stress by combining the stress components including; (1) stresses due to body forces $F_{x}=-\frac{\alpha(T) E(T)}{1-2 v(T)} \frac{\partial T}{\partial x^{\prime}} F_{z}=-\frac{\alpha(T) E(T)}{1-2 v(T)} \frac{\partial T}{\partial z}$ along the scan direction and build direction which can be obtained from;

$$
\begin{gathered}
\{\sigma\}=\int_{0}^{\infty} \int_{-\infty}^{\infty}\left(G\left(x, z, x^{\prime}, z^{\prime}\right) B d x^{\prime} d z^{\prime}\right. \\
\text { where }\{\sigma\}=\left\{\sigma_{x x} \sigma_{z z} \sigma_{x z}\right\}^{T} \\
G=\left(\begin{array}{cc}
G_{x h} & G_{x v} \\
G_{z h} & G_{z v} \\
G_{x z h} & G_{x z v}
\end{array}\right) \\
B=\left\{\begin{array}{l}
-\frac{\alpha(T) E(T)}{1-2 v(T)} \frac{\partial T}{\partial x} \\
-\frac{\alpha(T) E(T)}{1-2 v(T)} \frac{\partial T}{\partial z}
\end{array}\right\}
\end{gathered}
$$

As described by Saif et al. [28], "the elements of $\mathrm{G}$ represents the stresses in half plane due to an applied unit body force at $\left(x^{\prime}, z^{\prime}\right)$. For instance, $G_{x h}\left(x, z, x^{\prime}, z^{\prime}\right)$ is equal to the $\sigma_{x x}(x, z)$ due to the unit load action along the scan direction applied at $\left(x^{\prime}, z^{\prime}\right)$, whereas $G_{x v}\left(x, z, x^{\prime}, z^{\prime}\right)$ is equal to the $\sigma_{x x}(x, z)$ due to the unit load action in the transverse direction applied at $\left(x^{\prime}, z^{\prime}\right)^{\prime \prime}$. The elements of $\mathrm{G}$ are explained in Appendix.

(2) stress due to normal stress tension $N=\frac{\alpha(T) E(T) T}{1-2 v(T)}$ on the boundary ( $\left.\mathrm{z}=0\right)$. The normal stress $\sigma_{x x}$ due to the tension can be obtained from

$$
\sigma_{x x}=\frac{2}{\pi} \int_{-\infty}^{\infty} \frac{[(s-x) T-z N](s-x)^{2}}{\left((s-x)^{2}+z^{2}\right)^{2}} d s
$$

By putting temperature $(\mathrm{T}=0)$ and normal tension $N=\frac{\alpha(T) E(T) T}{1-2 v(T)}$, the integral reduces to

$$
\sigma_{x x}(x, z=0)=\frac{\alpha(T) E(T) T}{1-2 v(T)}
$$

(3) hydrostatic stress can be obtained as $-\frac{\alpha(T) E(T) T}{1-2 v(T)}$ as explained in the work of Cowper [29].

Accordingly, the stress due to the non-uniform heating in the build part is calculated by the combination of the different sources of stresses due to the body forces, normal stress tension, and hydrostatic stress as described by Saif et al. [28]: 


$$
\begin{gathered}
\sigma_{x x}(x, z)=-\frac{\alpha(T) E(T)}{1-2 v(T)} \int_{0}^{\infty} \int_{-\infty}^{\infty}\left(G_{x h} \frac{\partial T}{\partial x}\left(x^{\prime}, z^{\prime}\right)+G_{x v} \frac{\partial T}{\partial z}\left(x^{\prime}, z^{\prime}\right)\right) d x^{\prime} d z^{\prime} \\
+\frac{2 z}{\pi} \int_{-\infty}^{\infty} \frac{p(s)(s-x)^{2}}{\left((s-x)^{2}+z^{2}\right)^{2}} d s-\frac{\alpha(T) E(T) T(x, z)}{1-2 v(T)} \\
\sigma_{z z}(x, z)=-\frac{\alpha(T) E(T)}{1-2 v(T)} \int_{0}^{\infty} \int_{-\infty}^{\infty}\left(G_{z h} \frac{\partial T}{\partial x}\left(x^{\prime}, z^{\prime}\right)+G_{z v} \frac{\partial T}{\partial z}\left(x^{\prime}, z^{\prime}\right)\right) d x^{\prime} d z^{\prime} \\
+\frac{2 z^{3}}{\pi} \int_{-\infty}^{\infty} \frac{p(s)}{\left((s-x)^{2}+z^{2}\right)^{2}} d s-\frac{\alpha(T) E(T) T(x, z)}{1-2 v(T)} \\
\sigma_{x z}(x, z)=-\frac{\alpha(T) E(T)}{1-2 v(T)} \int_{0}^{\infty} \int_{-\infty}^{\infty}\left(G_{x z h} \frac{\partial T}{\partial x}\left(x^{\prime}, z^{\prime}\right)+G_{x z v} \frac{\partial T}{\partial z}\left(x^{\prime}, z^{\prime}\right)\right) d x^{\prime} d z^{\prime} \\
+\frac{2 z^{2}}{\pi} \int_{-\infty}^{\infty} \frac{p(s)(s-x)}{\left((s-x)^{2}+z^{2}\right)^{2}} d s \\
\sigma_{z z}(x, z)=v(T)\left(\sigma_{x x}+\sigma_{z z}\right)-\alpha(T) E(T) T(x, z)
\end{gathered}
$$

where, $\alpha$ is the coefficient of the thermal expansion, E represents the elastic modulus, $\frac{\partial T}{\partial x}$ is the temperature gradient and $p(s)$ is expressed by:

$$
p(s)=\frac{\alpha(T) E(T) T(x, z=0)}{1-2 v(T)}
$$

The closed-form solution of thermal stress can be derived as;

$$
\sigma_{x x}(i, j)=-\frac{\alpha E(T)}{1-2 v}\left(G_{x h} T_{x}+G_{x v} T_{z}\right)\left(\left|x_{i}-x_{i-1}\right|\left|z_{j}-z_{j-1}\right|\right)+\frac{\frac{2 z}{\pi}\left(p(s)(s-x)^{2}\right.}{\left((s-x)^{2}+z^{2}\right)^{2}}\left|x_{i}-x_{i-1}\right|-\frac{\alpha E T}{1-2 v}
$$

where $T_{x}=\frac{T(i, j)-\mathrm{T}(\mathrm{i}-1, \mathrm{j})}{\left|x_{i}-x_{i-1}\right|}, T_{z}=\frac{T(i, j)-\mathrm{T}(\mathrm{i}, \mathrm{j}-1)}{\left|z_{j}-z_{j-1}\right|}$ and $\mathrm{i}, \mathrm{j}$ represent a location in the $2 \mathrm{D}$ medium at which the stress is calculated. The closed-form solutions of $\sigma_{z z}$ and $\sigma_{x z}$ can be derived using the same method.

At elevated temperatures, the yield strength of the Ti-6Al-4V approaches zero. Accordingly, the AM part experiences a high magnitude of plastic deformation due to heating cycles and cooling cycles. If the stress passes the yield strength, upon unloading (cooling in this situation) some amount of stress remains in the body which is known as RS. The RS is inherent in all the AM parts and can be reduced or eliminated with proper control and optimization of process parameters or postprocessing techniques such as pitting, and heat treatment since it has an elastic nature.

Both the in-plane and out of plane RS distributions are obtained from incremental plasticity and kinematic hardening behavior of metal according to the conservation of volume in plastic deformation in coupling with equilibrium and compatibility conditions. McDowell et al. [30] proposed an algorithm to predict the RS in rolling. This algorithm has several advantages including high computational efficiency, rapid and accurate prediction of RS; however, this algorithm has several limitations; First, the stress along the build direction $\left(\sigma_{z z}\right)$ is assumed to be elastic. In AM processes, the stress along the build direction has a high magnitude. Thus, the stress along the build direction should be solved along with $\sigma_{x x}$, and $\sigma_{y y}$. In this case, there are two equations with three unknowns, which cannot be solved using McDowell model. Second, this algorithm does not consider the conservation of volume in plastic deformation. Qi et al. [31] explained that these limitations may reduce the precision of the predicted $\mathrm{RS}$.

To determine the yield surface, the Johnson-Cook method is used to model the flow stress as; 


$$
k=\frac{1}{\sqrt{3}}\left(A+B \varepsilon_{e f f}^{p}{ }^{n}\right)\left(1+C \ln \left(\frac{\dot{\varepsilon}_{\text {eff }}^{p}}{\dot{\varepsilon}_{0}}\right)\right)\left(1-\left[\frac{T-T_{0}}{T_{m}-T_{0}}\right]^{m}\right)
$$

where $k$ is material yield stress, $\varepsilon_{e f f}^{p}$ represents the effective plastic strain, $\dot{\varepsilon}_{e f f}^{p}$ is the effective plastic strain rate, $T$ is the temperature of material, $T_{m}$ is the melting point of material, and $T_{0}$ is the initial temperature. The terms $A, B, C, n, m$ and $\dot{\varepsilon}_{0}$ are the material constant which are listed in Table 1 for Ti-6Al-4V.

The effective plastic strain and strain rate are defined as

$$
\begin{gathered}
\varepsilon_{e f f}^{p}=\frac{\sqrt{2}}{3} \sqrt{\left(\varepsilon_{x x}^{p}-\varepsilon_{y y}^{p}\right)^{2}+\left(\varepsilon_{y y}^{p}-\varepsilon_{z z}^{p}\right)^{2}+\left(\varepsilon_{z z}^{p}-\varepsilon_{x x}^{p}\right)^{2}+6\left(\varepsilon_{x z}^{p}\right)^{2}} \\
\dot{\varepsilon}_{e f f}^{p}=\sqrt{\frac{2}{3}} \sqrt{\left(\dot{\varepsilon}_{x x}^{p}\right)^{2}+\left(\dot{\varepsilon}_{y y}^{p}\right)^{2}+\left(\dot{\varepsilon}_{z z}^{p}\right)^{2}+\left(\dot{\varepsilon}_{x z}^{p}\right)^{2}}
\end{gathered}
$$

The yielding criterion is obtained for an isotropic material. Kinematic hardening is considered by employing backstress tensor $\left(\alpha_{i j}\right)$

$$
\begin{gathered}
F_{\text {yeild }}=\frac{3}{2}\left(S_{i j}-\alpha_{i j}\right)\left(S_{i j}-\alpha_{i j}\right)-k^{2}=0 \\
\left\{\begin{array}{l}
F_{\text {yeild }}<0 \text { elastic deformation } \\
F_{\text {yeild }}>0 \text { plastic deformation }
\end{array}\right.
\end{gathered}
$$

where $S_{i j}=\sigma_{i j}-\left(\sigma_{k k} / 3\right) \delta_{i j}$ is the deviatoric stress, $k$ is the material yield threshold which is determined using material flow stress model.

$\dot{\alpha_{l j}}=\left\langle\dot{S_{k l}} n_{k l}\right\rangle n_{i j}$ shows the back stress tensor rate in linear kinematic hardening, where $<>$ is MacCauley bracket and is expressed as $\langle x\rangle=0.5(x+|x|)$, and $n_{i j}=\frac{s_{i j}-\alpha_{i j}}{\sqrt{2} k}$ which is the components of unit normal in plastic strain rate direction, and $k$ is the material flow stress threshold.

If $F_{\text {yeild }}<0$, material is in elastic region and the stresses can be obtained from the Hook's Law.

If $F_{\text {yeild }}>0$, the total plastic strains can be obtained by calculating the plastic strains incrementally during cyclic heating and cooling. The plastic strain rate is determined by Khan and Huang [32] as

$$
\dot{\varepsilon}_{i j}^{P}=\frac{1}{h}\left\langle\dot{S}_{k l} n_{k l}\right\rangle n_{i j}
$$

where $h$ is the plastic modulus. In the elastic-plastic case where the $F_{y e i l d} \geq 0$, the strain rate along the scan direction and transverse direction can be calculated using modified McDowell algorithm. In the elastoplastic loading the total strain is a combination of elastic part and plastic part as

$$
\begin{aligned}
\dot{\varepsilon}_{x x} & =\dot{\varepsilon}_{x x}{ }^{e}+\dot{\varepsilon}_{x x}{ }^{p} \\
\dot{\varepsilon}_{y y} & =\dot{\varepsilon}_{y y}{ }^{e}+\dot{\varepsilon}_{y y} p \\
\dot{\varepsilon}_{z z} & =\dot{\varepsilon}_{z z}{ }^{e}+\dot{\varepsilon}_{z z}{ }^{p}
\end{aligned}
$$

According to the conservation of volume in plastic deformation;

Based on plain strain assumption

$$
\dot{\varepsilon}_{x x}^{p}+\dot{\varepsilon}_{y y}^{p}+\dot{\varepsilon}_{z z}^{p}=0
$$

Then,

$$
\dot{\varepsilon}_{y y}{ }^{e}=\dot{\varepsilon}_{y y}^{p}=0
$$

$$
\dot{\varepsilon}_{x x}^{p}+\dot{\varepsilon}_{z z}^{p}=0
$$

Taking plastic flow rule (Equation (17)) into Equation (21); 
Based on plastic loading condition;

$$
\frac{1}{h}\left\langle S_{k l}^{\cdot} n_{k l}\right\rangle\left(n_{x x}+n_{z z}\right)=0
$$

$$
\left\langle\dot{S_{k l}} n_{k l}\right\rangle>0
$$

Therefore;

$$
\left(n_{x x}+n_{z z}\right)=0
$$

Taking $n_{i j}=\frac{s_{i j}-\alpha_{i j}}{\sqrt{2} k}$, and $S_{i j}=\sigma_{i j}-\left(\sigma_{k k} / 3\right) \delta_{i j}$ into Equation (22);

$$
\sigma_{y y}=\frac{1}{2}\left(\sigma_{x x}+\sigma_{z z}\right)
$$

The incremental form of Equation (25) can be written as;

$$
\dot{\sigma}_{y y}=\frac{1}{2}\left(\dot{\sigma}_{x x}+\dot{\sigma}_{z z}\right)
$$

Then, by solving three equations with three unknowns, $\left(\sigma_{x x}, \sigma_{y y}, \sigma_{z z}\right)$ can be obtained as;

$$
\left\{\begin{array}{c}
\frac{1}{E}\left[\dot{\sigma}_{x x}-v\left(\dot{\sigma}_{y y}-\dot{\sigma}_{z z}\right)\right]+\alpha \Delta T+\frac{1}{h}\left(\dot{\sigma}_{x x} n_{x x}+\dot{\sigma}_{y y} n_{y y}+\dot{\sigma}_{z z} n_{z z}+2 \dot{\sigma}_{x z}^{*} n_{x z}\right) n_{x x}= \\
\psi\left(\frac{1}{E}\left[\dot{\sigma}_{x x}^{*}-v\left(\dot{\sigma}_{y y}-\dot{\sigma}_{z z}^{*}\right)\right]+\alpha \Delta T+\frac{1}{h}\left(\dot{\sigma}_{x x}^{*} n_{x x}+\dot{\sigma}_{y y} n_{y y}+\dot{\sigma}_{z z}^{*} n_{z z}+2 \dot{\sigma}_{x z}^{*} n_{x z}\right) n_{x x}\right) \\
\frac{1}{E}\left[\dot{\sigma}_{y y}-v\left(\dot{\sigma}_{x x}-\dot{\sigma}_{z z}\right)\right]+\alpha \Delta T+\frac{1}{h}\left(\dot{\sigma}_{x x} n_{x x}+\dot{\sigma}_{y y} n_{y y}+\dot{\sigma}_{z z} n_{z z}+2 \dot{\sigma}_{x z}^{*} n_{x z}\right) n_{y y}=0 \\
\dot{\sigma}_{y y}=\frac{1}{2}\left(\dot{\sigma}_{x x}+\dot{\sigma}_{z z}\right)
\end{array}\right.
$$

where, $\dot{\sigma}_{x x}^{*}, \dot{\sigma}_{z z}^{*}, \dot{\sigma}_{x z}^{*}$ are the elastic thermal stresses calculated from Equations (9 and 10). $\psi$ is the hybrid function which depends on the modulus ratio $(h / G)$ as;

$$
\psi=1-\exp \left(-\xi \frac{3 h}{2 G}\right)
$$

where $\xi=0.15$ is the algorithm constant, $h$ is the plastic modulus, and $G=E /(2(1+v))$ is the elastic shear modulus. $\psi$ approaches zero as $h$ approaches zero (perfect plasticity), and $\psi$ approaches unity as $h$ approaches infinity (initial yielding). $\psi$ is always between unity and zero.

Three systems of equations are solved simultaneously for $\dot{\sigma}_{x x}, \dot{\sigma}_{y y}$, and $\dot{\sigma}_{z z}$ for each elasticplastic increment of strain.

Table 1. Johnson-Cook parameters for Ti-6Al-4V [33] .

\begin{tabular}{cccccc}
\hline $\mathrm{A}(\mathrm{MPa})$ & $\mathrm{B}(\mathrm{MPa})$ & $\mathrm{C}$ & $\mathrm{n}$ & $\mathrm{m}$ & $\dot{\boldsymbol{\varepsilon}}_{\mathbf{0}}$ \\
\hline $\mathbf{9 9 7 . 9}$ & 653.1 & 0.025 & 0.45 & 0.6 & 1 \\
\hline
\end{tabular}

After laser has scanned one layer, elastic stresses are relaxed to meet the boundary condition prescribed by Merwin and Johnson [34] as

$$
\begin{gathered}
\varepsilon_{x x}^{r}=0, \quad \sigma_{x x}^{r}=f_{1}(z), \quad \varepsilon_{y y}^{r}=0, \quad \sigma_{y y}^{r}=f_{2}(z), \quad \varepsilon_{z z}^{r}=f_{3}(z), \quad \sigma_{z z}^{r}=0, \\
\gamma_{x z}^{r}=f_{4}(z), \quad \sigma_{x z}^{r}=0
\end{gathered}
$$

Finally, only stresses and strains parallel to the surface $\left(\sigma_{x x}^{r}, \sigma_{y y}^{r}, \gamma_{x z}^{r}\right)$ remain non-zero. The only non-zero strain is $\varepsilon_{z z}^{r}$, resulting from surface compression. Accordingly, the non-zero components $\varepsilon_{x x}^{r}, \sigma_{z z}^{r}$, and $\sigma_{x z}^{r}$ at the end of each pass should incrementally relaxed to zero as;

$$
\Delta \sigma_{z z}=-\frac{\sigma_{z z}^{r}}{M}, \Delta \sigma_{x z}=-\frac{\sigma_{x z}^{r}}{M}, \Delta \varepsilon_{x x}=-\frac{\varepsilon_{x x}^{r}}{M}
$$

where $M$ is the number of increments (e.g.100-1000) for relaxation procedure.

Using Equation (29), for the case of purely elastic relaxation increment $(F \leq 0)$, the relaxation process is described by general Hook's law as 


$$
\left\{\begin{array}{l}
\Delta \sigma_{x x}=\frac{E \Delta \varepsilon_{x x}+(1+v)\left(\Delta \sigma_{z z} v-E \alpha \Delta T\right)}{\left(1-v^{2}\right)} \\
\Delta \sigma_{y y}=\frac{v E \Delta \varepsilon_{x x}+(1+v)\left(\Delta \sigma_{z z} v-E \alpha \Delta T\right)}{\left(1-v^{2}\right)}
\end{array}\right.
$$

$\Delta^{\prime} s$ replace the time derivative.

For the elastic-plastic case $(F>0)$, the released stresses calculated as

$$
\begin{gathered}
\left\{\begin{array}{c}
\Delta \sigma_{x x}=\frac{D-\left(\frac{1}{E}+\frac{1}{h} n_{x x} n_{y y}\right) \Delta \sigma_{y y}-\alpha \Delta \mathrm{T}}{-\frac{v}{E}+\frac{1}{h} n_{x x} n_{y y}} \\
\Delta \sigma_{y y}=\frac{\left(-\frac{v}{E}+\frac{1}{h} n_{x x} n_{y y}\right)(C-\alpha \Delta \mathrm{T})-\left(\frac{1}{E}+\frac{1}{h} n_{x x} n_{x x}\right)(D-\alpha \Delta \mathrm{T})}{\left[\left(-\frac{v}{E}+\frac{1}{h} n_{x x} n_{y y}\right)^{2}-\left(\frac{1}{E}+\frac{1}{h} n_{x x} n_{x x}\right)\left(\frac{1}{E}+\frac{1}{h} n_{y y} n_{y y}\right)\right]}
\end{array}\right. \\
\text { where }\left\{\begin{array}{c}
C=\Delta \varepsilon_{x x}+\left(\frac{v}{E}-\frac{1}{h} n_{x x} n_{z z}\right) \Delta \sigma_{z z}{ }^{*}-\frac{2}{h} \Delta \sigma_{x z}{ }^{*} n_{x z} n_{x x} \\
D=\left(\frac{v}{E}-\frac{1}{h} n_{y y} n_{z z}\right) \Delta{\sigma_{z z}}^{*}-\frac{2}{h} \Delta{\sigma_{x z}}^{*} n_{x z} n_{y y}
\end{array}\right.
\end{gathered}
$$

The residual stresses in the scan direction and build direction are then calculated as the remaining stresses after relaxation. Residual stress and stress relaxation algorithm are shown in Figure 3. 


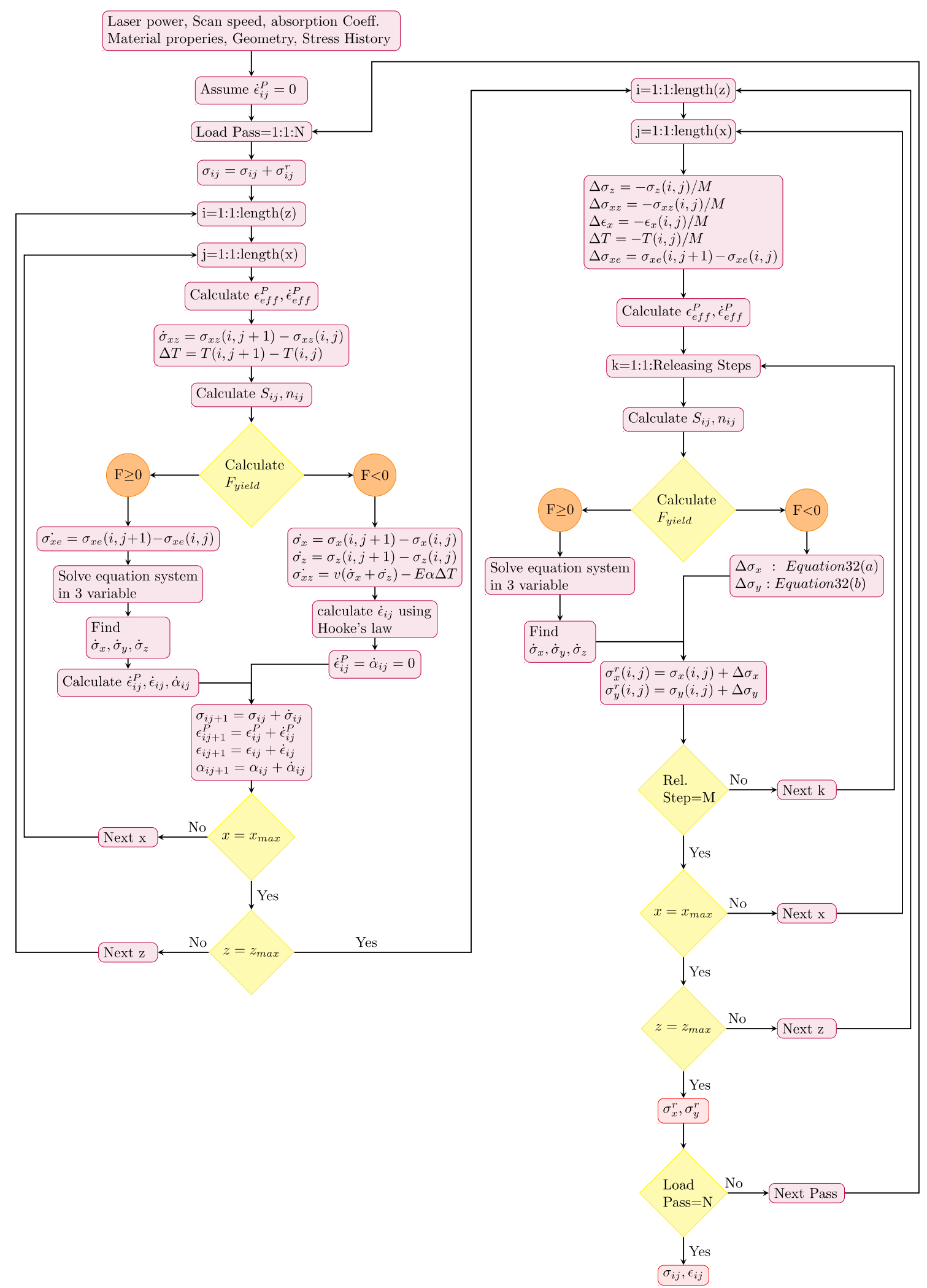

Figure 3. Residual stress and relaxation algorithms.

\section{Temperature Dependent Material Properties}

Due to the rapid heating and low conductivity, material experience steep temperature gradient. As a result of the steep temperature gradient, material properties could vary significantly in the medium. Therefore, it is crucial to consider material properties temperature dependent. Density, 
conductivity, specific heat, yield strength, Young's modulus, Poisson's ratio, and thermal expansion coefficient of Ti-6Al-4V are considered temperature-sensitive, as shown in Figure 4. These data are gathered from the work of Welsch et al. [35], Heigel et al. [36] Jamshidinia et al. [37], Li et al. [38] Mills[39], and Murgau [40]. The melting temperature range of Ti-6Al-4V is from $1600^{\circ} \mathrm{C}$ to $1660^{\circ} \mathrm{C}$. In this modeling melting temperature is $1600^{\circ} \mathrm{C}$. Density of Ti-6Al-4V drops for about $6.7 \%$ from room temperature to $2000^{\circ} \mathrm{C}$; thermal conductivity increases as the temperature increases; specific heat increases gradually with the increase in temperature; coefficient of thermal expansion increases gradually up to $1000^{\circ} \mathrm{C}$, and reaches a constant value; elastic modulus decreases with the increase in temperature and approaches zero at around melting temperature. This shows the material at liquid phase has negligible elasticity; Poisson's ratio increases with the increase in temperature; yield strength decreases rapidly from room temperature up to $1000^{\circ} \mathrm{C}$ and slows the slope above $1000^{\circ} \mathrm{C}$. This signifies that the Ti-6Al- $4 \mathrm{~V}$ is extremely soft and it is quite easy to undergo plastic deformation at elevated temperatures which causes the medium to experience high residual stress.

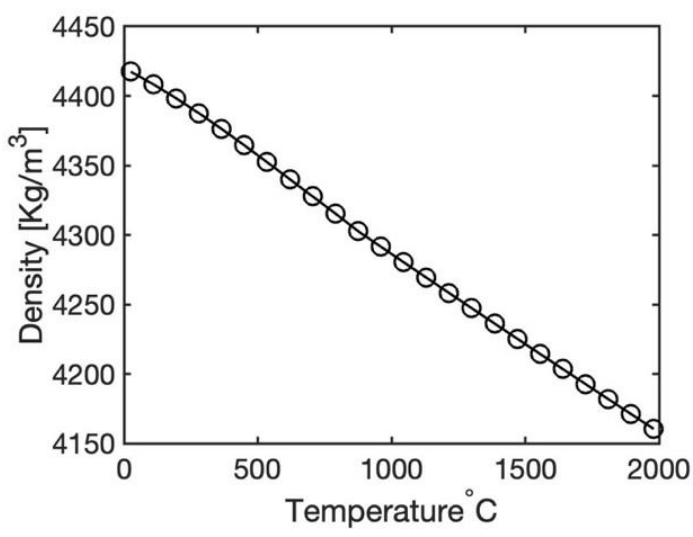

(a)

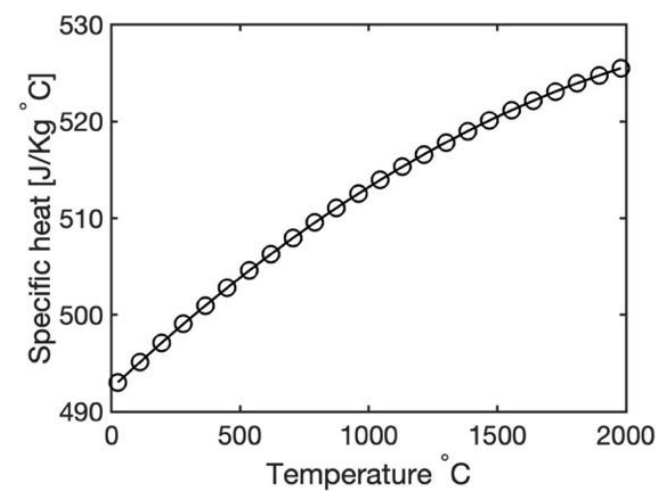

(c)

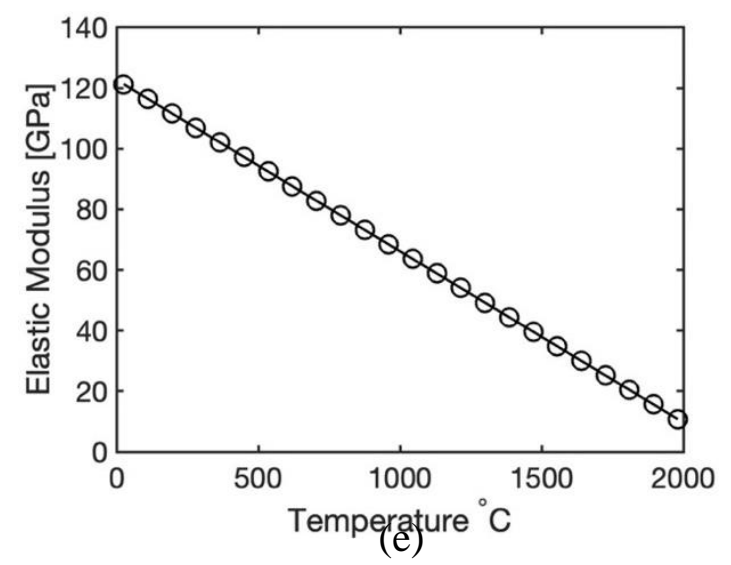

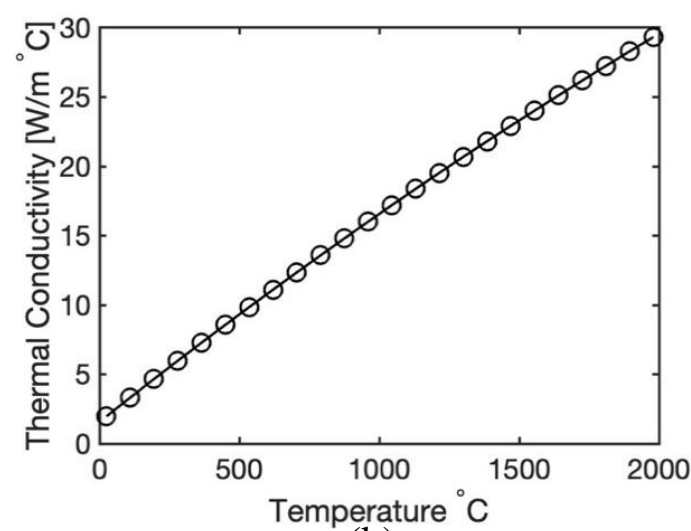

(b)
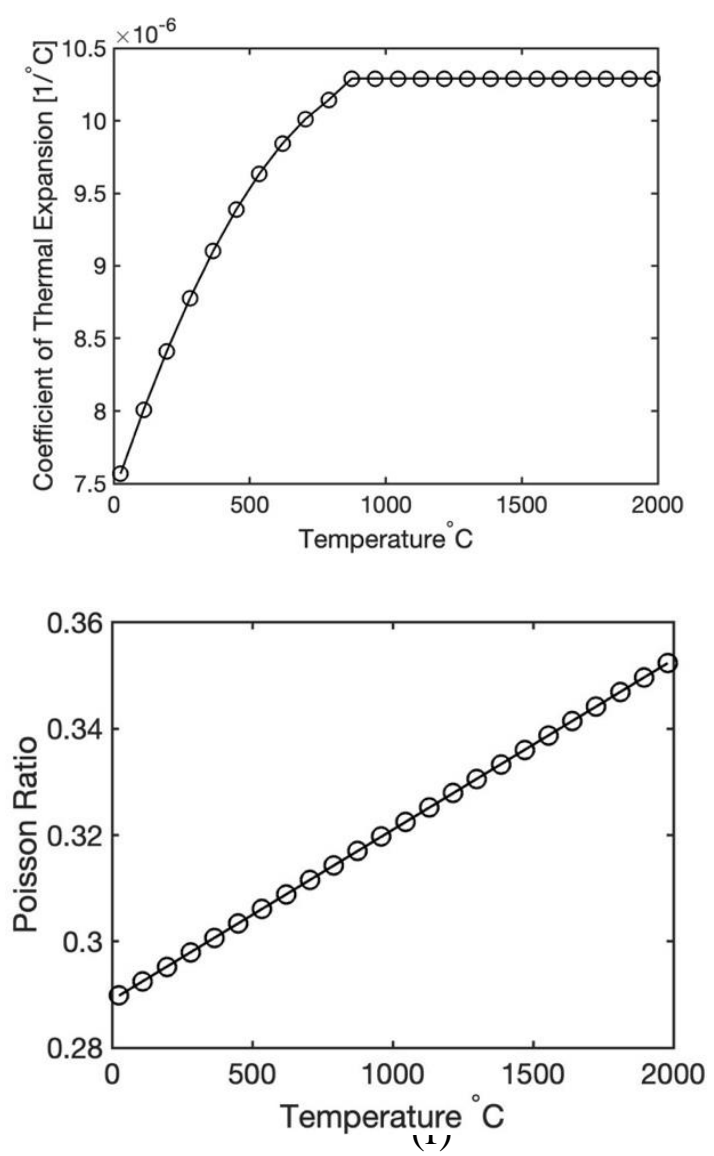


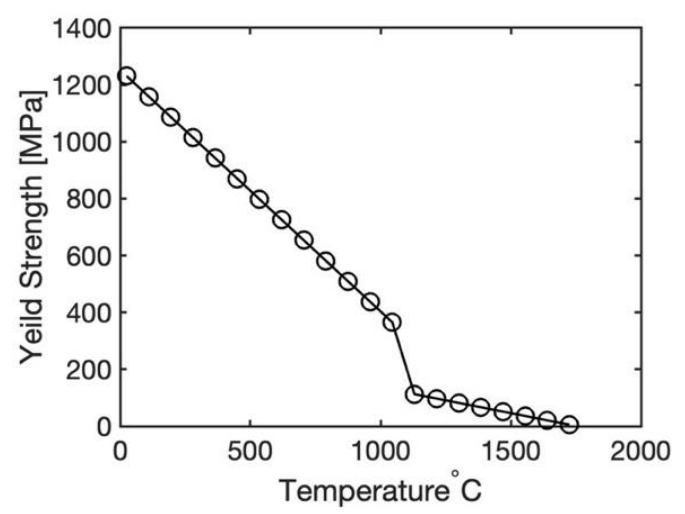

(g)

Figure 4. Temperature-dependent material properties of Ti-6Al-4V;(a) density;(b) thermal conductivity; (c) specific heat; (d) thermal expansion; (e) elastic modulus; (f) Poisson's ratio; (g) Yield strength

The equations of temperature sensitive material properties are also derived from the data points as listed in Table 2.

Table 2. Thermal and mechanical material properties of Ti-6Al-4V.

\begin{tabular}{|c|c|c|}
\hline Density $\left[\mathrm{kg} / \boldsymbol{m}^{3}\right]$ & $\rho=4420 /(1+\alpha \times T)^{3}$ & \\
\hline $\begin{array}{l}\text { Thermal conductivity } \\
{\left[\mathrm{W} / \mathrm{m}^{\circ} \mathrm{C}\right]}\end{array}$ & $K=1.57+1.6 e-2 \times T-1 e-6 \times T^{2}$ & \\
\hline Specific heat $\left[\mathrm{J} / \mathrm{kg}^{\circ} \mathrm{C}\right]$ & $C=492.4+0.025 \times T-4.18 e-6 \times T^{2}$ & \\
\hline $\begin{array}{l}\text { Thermal expansion } \\
{\left[1 /{ }^{\circ} \mathrm{C}\right]}\end{array}$ & $\left\{\begin{array}{l}\alpha=7.43 e-6+5.56 e-9 \times T-2.69 e-12 \times T^{2} \\
\alpha=10.291 e-6\end{array}\right.$ & $\begin{array}{l}T<827 \\
T>827\end{array}$ \\
\hline Elastic modulus [GPa] & $E=122.7-0.0565 \times T$ & \\
\hline Poisson's ratio & $v=0.289+3.2 e-5 \times T$ & \\
\hline Yield strength [MPa] & $\left\{\begin{array}{l}\sigma_{Y}=1256-0.8486 \times T \\
\sigma_{Y}=316-0.16 \times T\end{array}\right.$ & $\begin{array}{r}T<1127 \\
T>1127\end{array}$ \\
\hline
\end{tabular}

\section{Experimental Residual Stress Analysis}

Two blocks of Ti-6Al-4V specimens with the size of $20 \times 10 \times 3 \mathrm{~mm}$ is produced via DMD process using LENS CS 1500 SYSTEMS under different process conditions as shown in Table 3. The selected laser powers are $206 \mathrm{~W}$, and $385 \mathrm{~W}$, and the scan speeds are $25 \mathrm{~mm} / \mathrm{s}, 40 \mathrm{~mm} / \mathrm{s}$, respectively. The deposited layer thickness for both samples is $250 \mu \mathrm{m}$, and hatch spacing is $105 \mu \mathrm{m}$. A bi-directional continuous scan strategy is used to build the parts as shown in Figure 2.

Table 3. Process parameters for DMD of Ti-6Al-4V specimens.

\begin{tabular}{lrlllc}
\hline $\begin{array}{l}\text { laser power } \\
(\mathrm{W})\end{array}$ & $\begin{array}{r}\text { Scan } \\
(\mathrm{mm} / \mathrm{s})\end{array}$ & speed & $\begin{array}{l}\text { Feed rate } \\
(\mathrm{gram} / \mathrm{s})\end{array}$ & $\begin{array}{l}\text { layer height } \\
(\boldsymbol{\mu m})\end{array}$ & $\begin{array}{l}\text { Hatch spacing } \\
(\boldsymbol{\mu m})\end{array}$ \\
\hline 206 & 25 & 1 & 250 & 105 \\
\hline 385 & 40 & 0.5 & 250 & 105 \\
\hline
\end{tabular}

PANalytical Empyrean multipurpose X-ray diffractometer is used to measure the RS of the specimens using the $\sin ^{2} \Psi$ method [41, 42]. The residual strains are determined as

$$
\varepsilon=\frac{d-d_{0}}{d_{0}}
$$

where, $d$ and $d_{0}$ are the stressed and unstressed lattice parameter, respectively.

The generalized Hook's law for isotropic material is used to calculate stress as

$$
\sigma_{i}=\frac{E}{(1+v)(1-2 v)}\left((1-v) \varepsilon_{i}+v\left(\varepsilon_{j}+\varepsilon_{k}\right) \quad \text { where } i, j, k \in x, y, z\right.
$$


In Equation (35), an elastic modulus $(E)$, and Poisson's ratio of $114 \mathrm{GPa}$ and 0.33 are used, respectively. Samples are polished using liquid abrasive of $1 \mu \mathrm{m}$ and $0.05 \mu \mathrm{m}$ at a very slow speed to eliminate macroscopic residual stresses. Measurements are collected every $0.5 \mathrm{~mm}$ along the build direction (z-axis) of the samples.

\section{Results and Discussion}

Figure 5 illustrates the melt pool area and heat affected zone induced by highly localized heat input and low thermal conductivity. Non-uniform heating induces non-uniform thermal expansion. During heating cycle, the melt pool area and the heat affected zone are trying to expand; however, they are surrounded by solidified metal. This would generate the compressive stress state within the melt pool and heat-affected zones as shown in Figure 6(a). During the cooling cycle, the shrinkage of the material would develop tensile stress state as illustrated in Figure 6(b).

The proposed analytical model is extremely valuable since it provides fast (less than 45 seconds with 4-processor laptop) and accurate prediction of stress state within the build. In this modeling, first, a transient moving heat source approach is used to predict the temperature field in AM. Second, the thermal stress induced is calculated by combining three stresses known as stresses due to body forces, normal tension, and hydrostatic stress. Last, the stresses may exceed the yield point and material would experience plastic deformation. As a consequence of repeated loading and unloading (heating and cooling), material experiences high RS. Therefore, both the in plane and out of plane RS distributions are calculated from incremental plasticity and kinematic hardening behavior of the metal based upon the premises of plane strain condition in the build of isotropic and homogeneous properties, in coupling with the equilibrium and compatibility conditions.

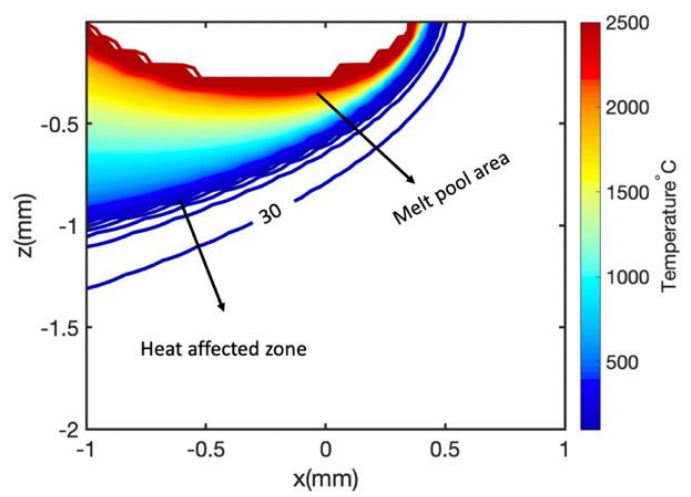

Figure 5. Illustration of melt pool and heat-affected zone.

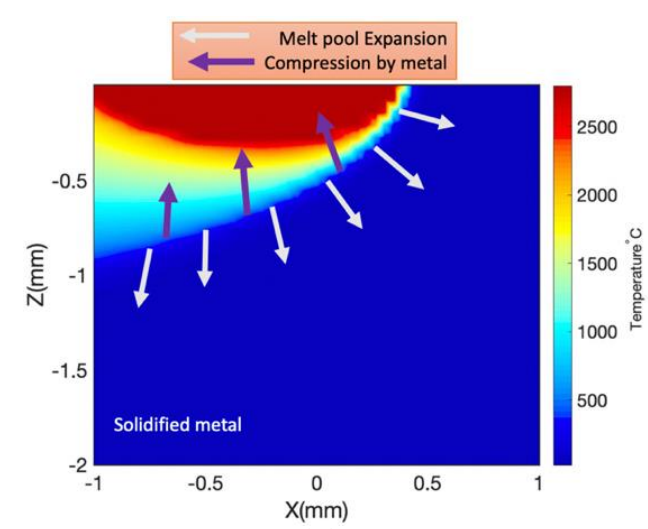

(a)

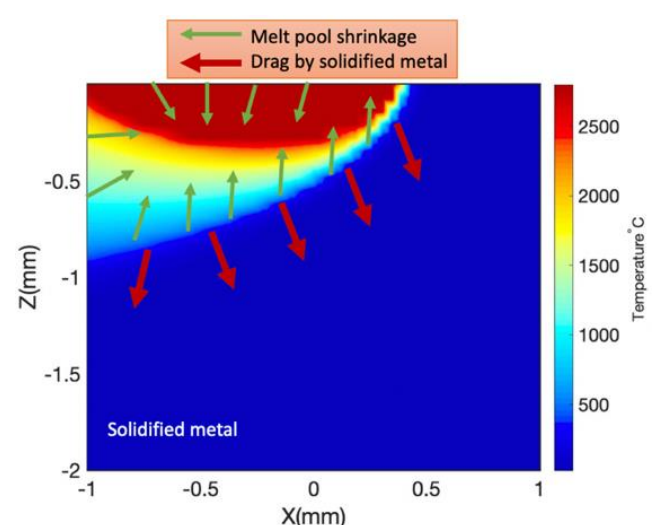

(b)

Figure 6. Illustration of accumulation of stress during (a) heating cycle; (b) cooling cycle

Material properties could vary significantly from point to point due to the steep temperature gradient. Consequently, it is not a fair assumption to consider the material properties constant. Herein, the thermal and mechanical material properties are temperature sensitive. Moreover, in this 
process, material experience cyclic melting and solidification. The energy needed for the phase change is taken into account by incorporating the latent heat of fusion into specific heat. Furthermore, the multi-layer and multi-scan aspect of metal AM are considered by including the temperature histories from the previous layers and scans.

Experimental measurements are conducted via $\mathrm{X}$-ray diffraction to measure the RS in Ti-6Al-4V specimens built via DMD process at the middle of the specimens $(X=10 \mathrm{~mm}, \mathrm{Y}=1.5 \mathrm{~mm})$ along the build direction (z-axis) at every $0.5 \mathrm{~mm}$. The scan strategy in both experimentation and analytical modeling is bi-directional. Moreover, the hatching space and layer thickness are $105 \mu \mathrm{m}$, $250 \mu \mathrm{m}$, respectively. Comparison of the results from the proposed analytical model and experimentation of Ti-6Al-4V specimens built via DMD process showed good qualitative and quantitative agreement.

Figure 7 illustrates the predicted temperature field for Ti-6Al-4V specimens. Figure 7(a) demonstrates the predicted melt pool area and heat-affected zone (HAZ) for the first specimen in Table 3, which has a laser power of $206 \mathrm{~W}$, the scan speed of $25 \mathrm{~mm} / \mathrm{s}$, with the layer height and hatch spacing of $250 \mu \mathrm{m}$ and $105 \mu \mathrm{m}$, respectively. It should be noted that the absorption ratio is $30 \%$ for Ti-6Al-4V samples as explained in the work of Selvan et al. [43]. Since the evaporation of the metallic powders is not considered in the modeling, the maximum temperature does not go beyond the evaporation temperature which is around $3000^{\circ} \mathrm{C}$ for Ti-6Al- $4 \mathrm{~V}$ as reported by Selvan et al. [43]. In this figure, the melt pool depth is around $0.1 \mathrm{~mm}$ based on the melting point of $1600^{\circ} \mathrm{C}$, and the depth of heat affected zone is around $0.15 \mathrm{~mm}$. Below this depth, the material is not affected by laser. The rapid temperature change at the border of HAZ and complete solid material- which is not affected by the laser- is the region where the stress state within the build part changes from tensile to compressive. This phenomenon will be explained in detail in the following sections of this manuscript. Figure 7(b) illustrates the predicted temperature field for the second specimen with the laser power of $385 \mathrm{~W}$, scan speed of $40 \mathrm{~mm} / \mathrm{s}$, layer height of $250 \mu \mathrm{m}$ and hatch spacing of $105 \mu \mathrm{m}$. Based on the melting point of Ti- $6 \mathrm{Al}-4 \mathrm{~V}\left(1600^{\circ} \mathrm{C}\right)$, the melt pool depth is $0.14 \mathrm{~mm}$ and the HAZ depth is around $0.21 \mathrm{~mm}$. The proposed temperature model is validated in the previous work of Mirkoohi et al. [24].

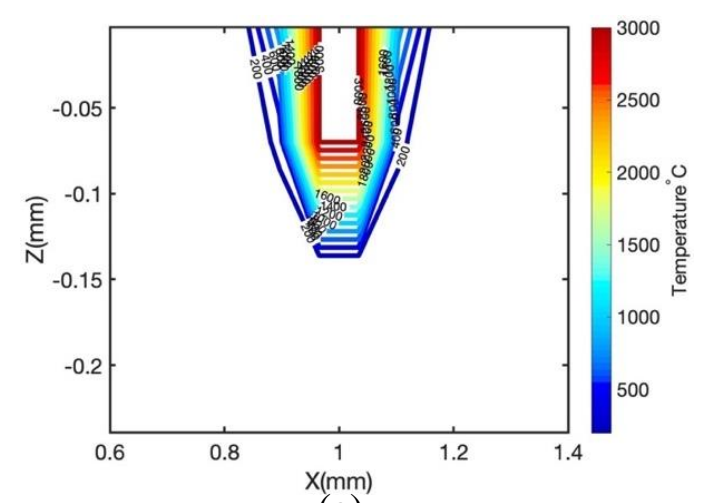

(a)

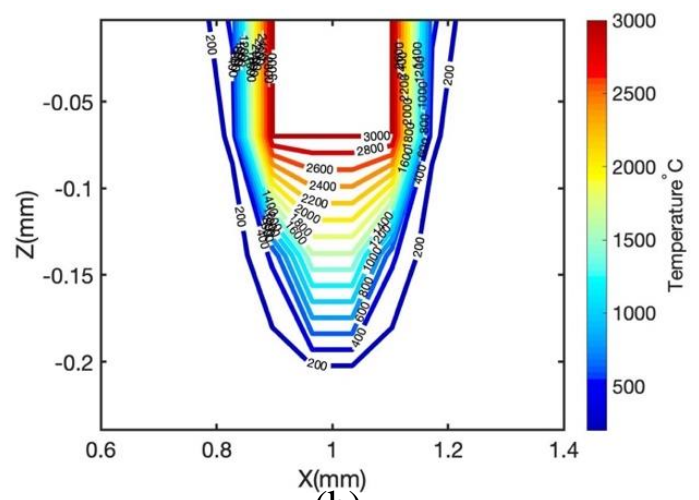

$(\mathrm{b})$

Figure 7. Predicted temperature field for Ti-6Al-4V with (a) laser power $=206 \mathrm{~W}$ and scan speed $=25$ $\mathrm{mm} / \mathrm{s}$; (b) laser power= $385 \mathrm{~W}$ and scan speed $=40 \mathrm{~mm} / \mathrm{s}$.

As explained before material properties vary significantly since the temperature gradient is quite high in AM. The variation of thermal and mechanical material properties for the second specimen with the laser power of $385 \mathrm{~W}$, and scan speed of $40 \mathrm{~mm} / \mathrm{s}$ illustrated in Figure 8. Density of the solid material is around $4400 \mathrm{~kg} / \mathrm{m}^{3}$. As the temperature increase the density decreases to $4050 \mathrm{~kg} / \mathrm{m}^{3}$ in the liquid zone; in the melt pool zone, thermal conductivity reaches its maximum value due to the high magnitude of temperatures, and decreases to around $5 \mathrm{~W} / \mathrm{m}^{\circ} \mathrm{C}$ in the solid zone; specific heat has the highest magnitude of $530 \mathrm{~J} / \mathrm{Kg}^{\circ} \mathrm{C}$ at the melt pool area and drops to $480 \mathrm{~J} / \mathrm{Kg}^{\circ} \mathrm{C}$ when the material is solidified; thermal expansion is more expanded in the liquid zone compare to other properties which shows the thermal expansion is more sensitive to temperature, and has the highest magnitude in the liquid zone; elastic modulus in the liquid zone is almost zero, in the HAZ reaches 
to $60 \mathrm{GPa}$, and in the solid zone reaches the maximum value of $120 \mathrm{GPa}$; Poisson's ratio in the liquid zone has the magnitude of 0.38 , and in the solid zone has the value of 0.29 ; yield strength reaches to almost zero at liquid zone and has the maximum value of $1200 \mathrm{MPa}$ in solid zone.

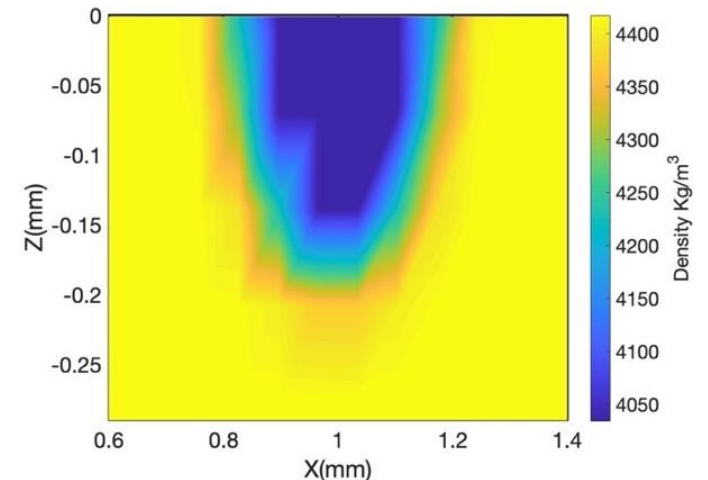

(a)

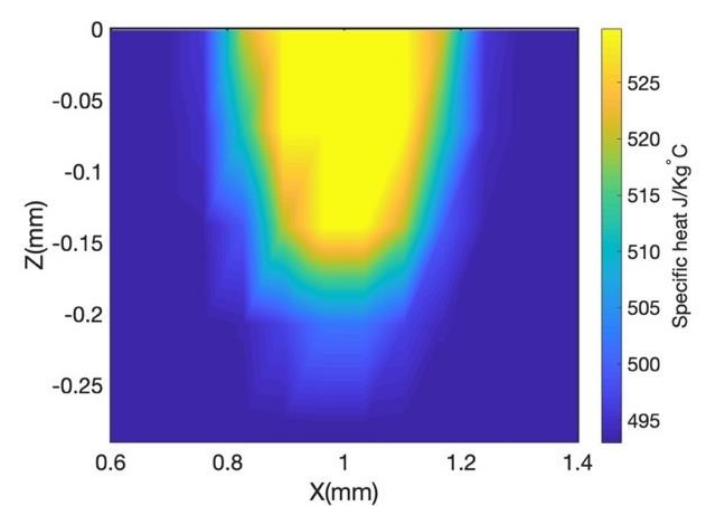

(c)

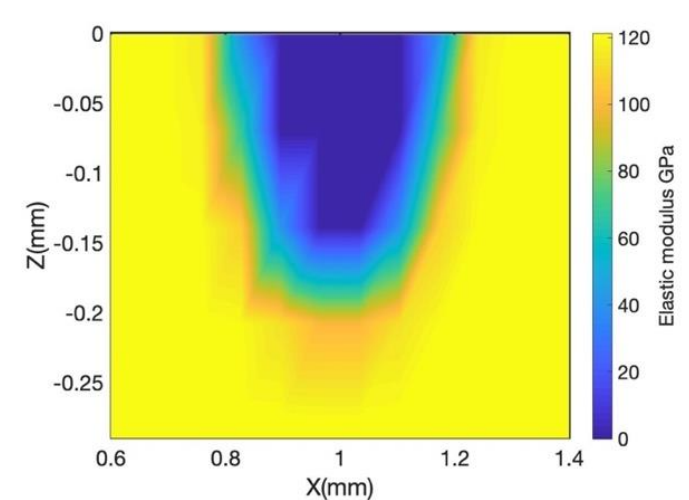

(e)

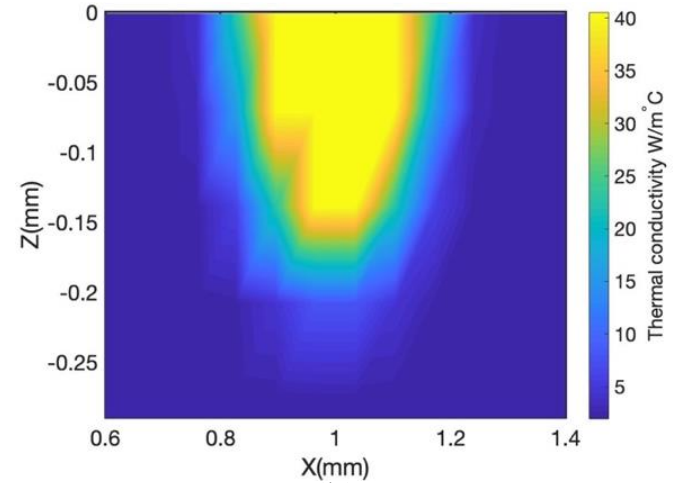

(b)

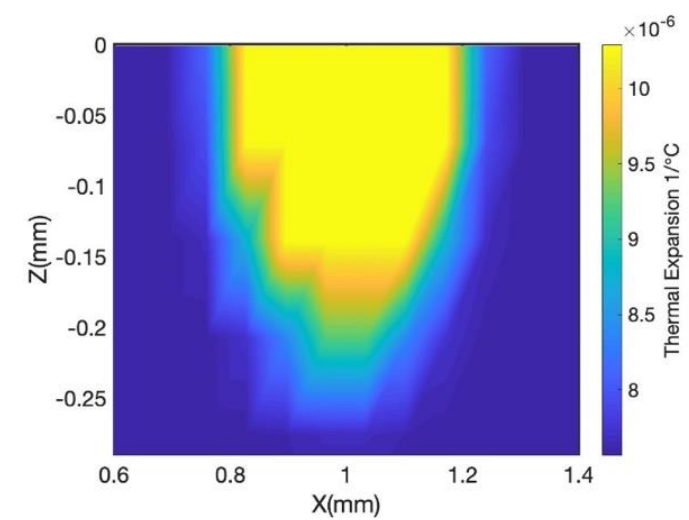

(d)

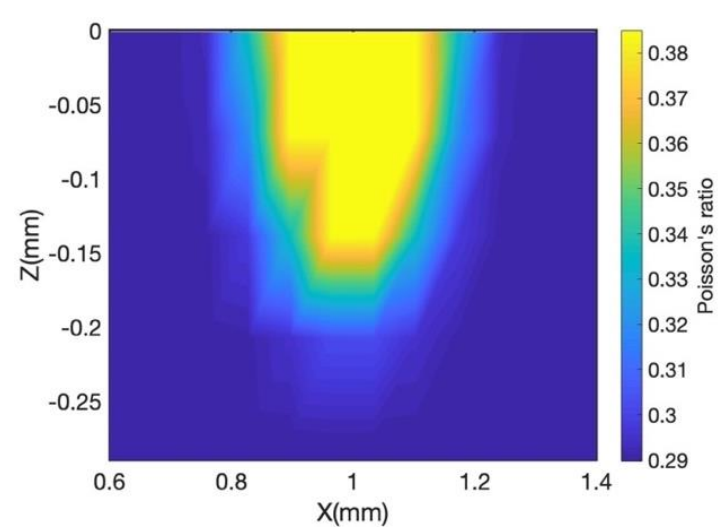

(f) 


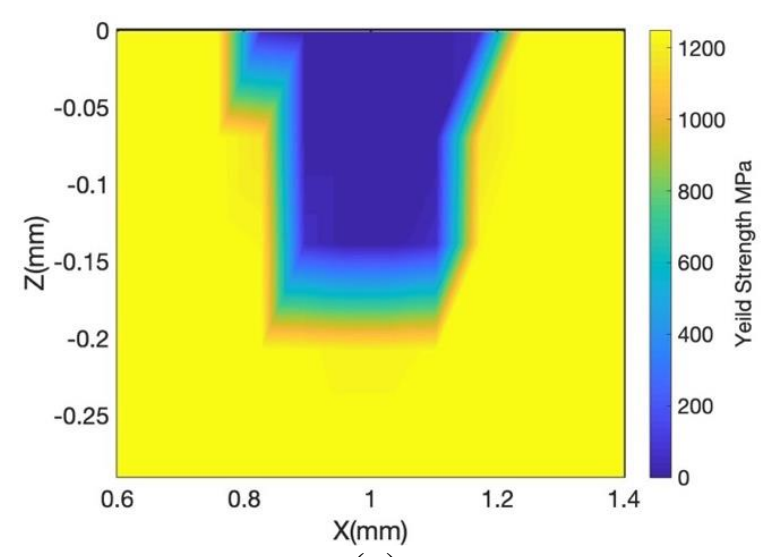

$(\mathrm{g})$

Figure 8. Predicted material properties distribution for Ti-6Al-4V with the laser power of $385 \mathrm{~W}$, and scan speed of $40 \mathrm{~mm} / \mathrm{s}$. (a) density;(b) conductivity;(c) specific heat; (d) thermal expansion; (e) elastic modulus; (f)

Poisson's ratio; $(\mathrm{g})$ Yield strength

Residual stress along the scan direction and transverse direction is predicted using the proposed model and validated experimentally. Figure 9 illustrates predicted RS along the scan direction and transverse direction as a function of depth into the build part for the first sample with the laser power of $206 \mathrm{~W}$, scan speed of $25 \mathrm{~mm} / \mathrm{s}$, layer height of $250 \mu \mathrm{m}$, and hatch spacing of and $105 \mu \mathrm{m}$. Since the samples have the rough surface, the top surface of the samples is polished up to $100 \mu \mathrm{m}$ with the electromechanical polishing to be able to accurately measure the RS on the surface.

Both the in plane and out of plane residual stresses are highly tensile in the melt pool zone and heat affected zone in coherence with most of reported results in literature as explained in introduction section. As shown in Figures 9 and 10, in both scan direction and transverse direction, there is a gradual change in stress state from tensile to compressive. This change occurs at around the depth where the medium is solidified and is not affected by the laser. The results confirm that upon cooling the melt pool and heat affected zones are under tension due to the shrinkage of the material, and when the below material is completely solidified and is not affected by the laser anymore, material experience compression stress state. The oscillations in the melt pool and heat affected zone is due to the fact that the thermal and mechanical material properties vary significantly in these regions. Therefore, an abrupt change in one of the material properties would results in oscillations. This could be better improved by having more material properties data points at different temperatures.

Figure 10 shows the predicted RS in the scan and transverse directions for the second sample in Table 2 with the laser power of $385 \mathrm{~W}$, scan speed of $25 \mathrm{~mm} / \mathrm{s}$, and layer height and hatch spacing of $250 \mu \mathrm{m}$ and $105 \mu \mathrm{m}$, respectively. The stress state is tensile in both the scan and transverse directions and changes to compressive at the depth around $0.9 \mathrm{~mm}$. Around this region the material is completely solidified and is not affected by the laser. Comparison of predicted and measured RS follows each other closely.

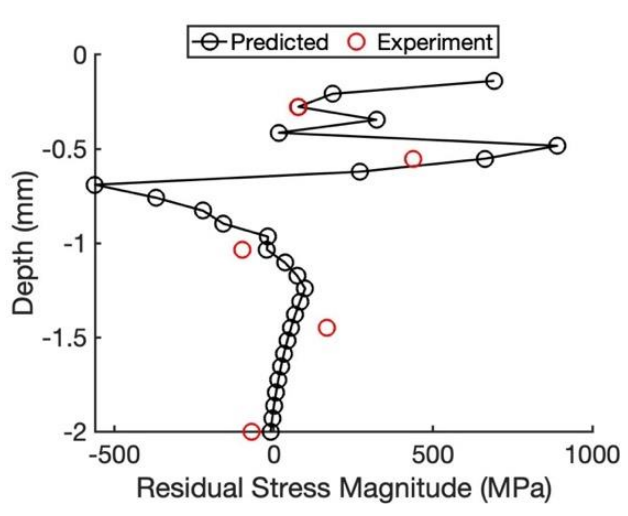

(a)

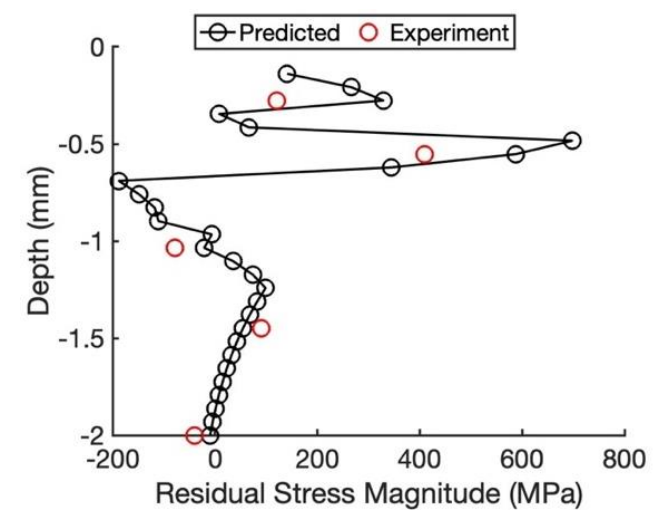

(b) 
Figure 9. Predicted residual stress for Ti-6Al-4V with laser power of $206 \mathrm{~W}$, and scan speed of $25 \mathrm{~mm} / \mathrm{s}$ along (a) scan direction; (b) transverse direction.

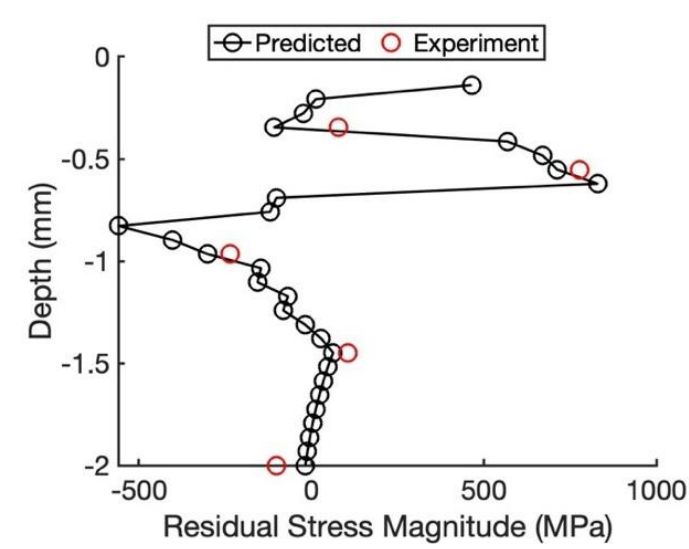

(a)

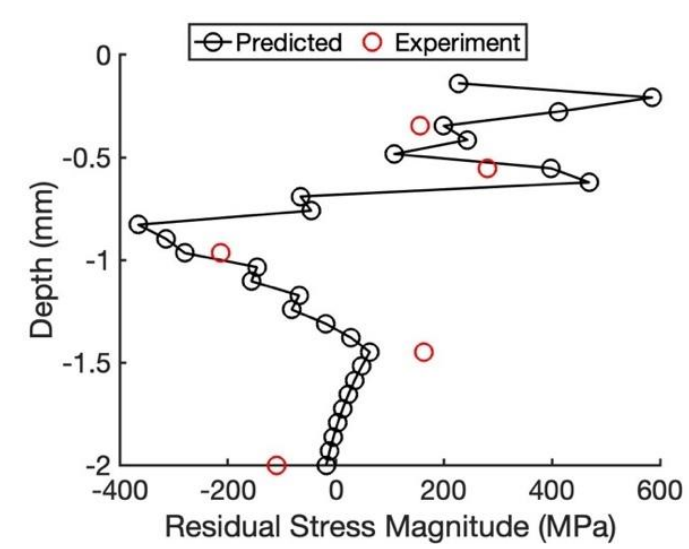

(b)

Figure 10. Predicted residual stress for Ti-6Al-4V with laser power of $385 \mathrm{~W}$, and scan speed of $40 \mathrm{~mm} / \mathrm{s}$ along (a) scan direction; (b) transverse direction.

\section{Conclusion}

A physics-based analytical model is proposed to rapidly and accurately calculate the stress state within the additively manufactured part. As the explicit computation is executed without meshing or iteration, it can be completed at a speed several orders of magnitude higher than that of conventional FEM's. The high computational efficiency of the proposed model affects a wide range of applications becoming a powerful tool for design and also fatigue assessment when component undergoes cyclic loading. It also enables efficient control and optimization of process parameters to achieve a high-quality part.

In this modeling, a transient moving point heat source is employed to predict the in-process temperature field within the build part. Thermal stress induced by steep temperature gradient is then obtained using the Green's functions of stresses due to the point body load in a homogeneous semiinfinite medium. Last, the stress state is obtained by importing the thermal history as an input for residual stress prediction using modified McDowell algorithm, in which both the in plane and out of plane residual stress distributions are obtained from incremental plasticity and kinematic hardening behavior of the metal, in coupling with the equilibrium and compatibility conditions. In this modeling, the relationship between three normal stresses are considered according to the property of volume invariance in plastic deformation.

Due to the fast irradiation of the laser and quite low thermal conductivity of the material, the material experience high temperature gradient. High temperature gradient induced variation of the material properties in the medium. Consequently, material properties are considered temperature sensitive. Moreover, the energy needed for the solid-state phase change is considered by modifying the heat capacity using latent heat of fusion. Furthermore, the effect of layer thickness, hatching space and scan strategy is considered in this modeling by incorporating the temperature history from the previous scans.

In this work, first, the temperature field is predicted for Ti-6Al-4V specimens. Distribution of thermal and mechanical material properties including density, specific heat, thermal conductivity, elastic modulus, coefficient of thermal expansion, yield strength, and Poisson's ratio for the laser power of $385 \mathrm{~W}$ and scan speed of $40 \mathrm{~mm} / \mathrm{s}$ showed that high temperature gradient in the AM process changes the material properties within the medium notably.

The predicted temperature fields for both samples showed that at the vicinity of melt pool area the temperature and the magnitude of temperature gradient is high enough to cause the thermal stress to exceed the yield strength of material. Upon cyclic loading and unloading of material during the heating and cooling cycles, material experience high residual stress. The predicted residual stress showed that the stress state is highly tensile in the melt pool and heat affected zones, and compressive 
in the solidified regions. Good qualitative and quantitative agreement is achieved between predicted and measured residual stress for Ti-6Al-4V samples.

Author Contributions: For research articles with several authors, a short paragraph specifying their individual contributions must be provided. The following statements should be used "Conceptualization, E.M.; methodology, E.M.; software, E.M.; validation, E.M.; formal analysis, E.M.; investigation, E.M.; resources, E.M., S.L., D.L., H.G.; data curation, E.M.; writing-original draft preparation, E.M.; writing-review and editing, E.M., S.L.; visualization, E.M; supervision, S.L. All authors have read and agreed to the published version of the manuscript.

Funding: This research received no external funding

Conflicts of Interest: The authors declare no conflict of interest.

\section{Appendix A}

Elements of $\mathrm{G}$ matrix

Let the $X_{m}=x-x^{\prime}, Z_{P}=z+z^{\prime}, Z_{m}=z-z^{\prime}$,

As explained by Saif et al. [28], "the Green functions $G_{x h}\left(x, z, x^{\prime}, z^{\prime}\right)$ and $G_{x v}\left(x, z, x^{\prime}, z^{\prime}\right)$ are the normal stress due to a unit point body load acting at $\left(x^{\prime}, z^{\prime}\right)$ along the $\mathrm{x}$ and $\mathrm{z}$ directions, respectively".

$$
\begin{aligned}
& G_{x h}=\frac{1}{4 \pi} \frac{1-2 v^{\prime}}{1-v^{\prime}}\left[\frac{3-2 v^{\prime}}{1-2 v^{\prime}}\left(\frac{X_{m}}{X_{m}^{2}+Z_{p}^{2}}-\frac{X_{m}}{X_{m}^{2}+Z_{m}^{2}}\right)+\frac{2}{1-2 v^{\prime}}\left(\frac{X_{m} Z_{m}^{2}}{\left(X_{m}^{2}+Z_{m}^{2}\right)^{2}}-\frac{X_{m} Z_{p}^{2}}{\left(X_{m}^{2}+Z_{p}^{2}\right)^{2}}\right)\right] \\
& -\frac{1}{\pi} \frac{1}{1-v^{\prime}} \times\left(\left(3-2 v^{\prime}\right) \frac{X_{m}\left(z^{\prime} Z_{p}+X_{m}^{2}\right)}{\left(X_{m}^{2}+Z_{p}^{2}\right)^{2}}-\frac{3 z^{\prime 2} X_{m} Z_{p}^{2}+X_{m}^{3}\left(4 z^{\prime 2}+6 z z^{\prime}+z^{2}+X_{m}^{2}\right)}{\left(X_{m}^{2}+Z_{p}^{2}\right)^{3}}\right) \\
& G_{z h}=-\frac{1}{4 \pi} \frac{1-2 v^{\prime}}{1-v^{\prime}}\left[\left(\frac{X_{m}}{X_{m}^{2}+Z_{p}^{2}}-\frac{X_{m}}{X_{m}^{2}+Z_{m}^{2}}\right)+\frac{2}{1-2 v^{\prime}}\left(\frac{X_{m} Z_{m}^{2}}{\left(X_{m}^{2}+Z_{m}^{2}\right)^{2}}-\frac{X_{m} Z_{p}^{2}}{\left(X_{m}^{2}+Z_{p}^{2}\right)^{2}}\right)\right] \\
& -\frac{1}{\pi} \frac{y}{1-v^{\prime}} \times\left(\left(3-2 v^{\prime}\right) \frac{X_{m} Z_{p}}{\left(X_{m}^{2}+Z_{p}^{2}\right)^{2}}-\frac{X_{m}\left(4 z^{\prime 3}+9 z z^{\prime 2}+6 z^{2} z^{\prime}+z^{3}+z X_{m}^{2}\right.}{\left(X_{m}^{2}+Z_{p}^{2}\right)^{3}}\right) \\
& G_{x z h}=\frac{1}{4 \pi} \frac{1-2 v^{\prime}}{1-v^{\prime}}\left[\left(\frac{Z_{P}}{X_{m}^{2}+Z_{p}^{2}}-\frac{Z_{m}}{X_{m}^{2}+Z_{m}^{2}}\right)+\frac{2}{1-2 v^{\prime}}\left(\frac{Z_{p} X_{m}^{2}}{\left(X_{m}^{2}+Z_{p}^{2}\right)^{2}}-\frac{Z_{m} X_{m}^{2}}{\left(X_{m}^{2}+Z_{m}^{2}\right)^{2}}\right)\right] \\
& -\frac{1}{\pi} \frac{1}{1-v^{\prime}} \\
& \times\left(\left(3-2 v^{\prime}\right) \frac{z^{\prime} Z_{p}^{2}+X_{m}^{2}\left(2 z+z^{\prime}\right)}{2\left(X_{m}^{2}+Z_{p}^{2}\right)^{2}}\right. \\
& \left.-\frac{z^{\prime 3}\left(z^{\prime 2}+3 z z^{\prime}+3 z^{2}\right)+z^{3} z^{\prime 2}+X_{m}^{2}\left(z^{\prime 3}+6 z z^{\prime 2}+6 z^{2} z^{\prime}+z^{3}\right)+z X_{m}^{4}}{\left(X_{m}^{2}+Z_{p}^{2}\right)^{3}}\right) \\
& G_{x v}=-\frac{1}{4 \pi} \frac{1-2 v^{\prime}}{1-v^{\prime}}\left[\left(\frac{Z_{P}}{X_{m}^{2}+Z_{p}^{2}}-\frac{Z_{m}}{X_{m}^{2}+Z_{m}^{2}}\right)+\frac{2}{1-2 v^{\prime}}\left(\frac{Z_{m} X_{m}^{2}}{\left(X_{m}^{2}+Z_{m}^{2}\right)^{2}}-\frac{Z_{p} X_{m}^{2}}{\left(X_{m}^{2}+Z_{p}^{2}\right)^{2}}\right)\right] \\
& -\frac{1}{2 \pi} \frac{1}{1-v^{\prime}} \\
& \times\left(2\left(1-v^{\prime}\right) \frac{Z_{p}}{\left(X_{m}^{2}+Z_{p}^{2}\right)}-\frac{\left[2\left(1-v^{\prime}\right) z-z^{\prime}\right]\left(Z_{p}^{2}-X_{m}^{2}\right)}{\left(X_{m}^{2}+Z_{p}^{2}\right)^{2}}+\frac{2 z z^{\prime} Z_{p}\left(3 X_{m}^{2}-Z_{p}^{2}\right)}{\left(X_{m}^{2}+Z_{p}^{2}\right)^{3}}\right) \\
& G_{z v}=\frac{1}{4 \pi} \frac{1}{1-v^{\prime}}\left[\left(3-2 v^{\prime}\right)\left(\frac{Z_{P}}{X_{m}^{2}+Z_{p}^{2}}-\frac{Z_{m}}{X_{m}^{2}+Z_{m}^{2}}\right)+2\left(\frac{Z_{m} X_{m}^{2}}{\left(X_{m}^{2}+Z_{m}^{2}\right)^{2}}-\frac{Z_{p} X_{m}^{2}}{\left(X_{m}^{2}+Z_{p}^{2}\right)^{2}}\right)\right] \\
& -\frac{1}{2 \pi} \frac{1}{1-v^{\prime}} \\
& \times\left(2\left(1-v^{\prime}\right) \frac{Z_{p}}{\left(X_{m}^{2}+Z_{p}^{2}\right)}+\frac{\left[2\left(1-v^{\prime}\right) z+z^{\prime}\right]\left(Z_{p}^{2}-X_{m}^{2}\right)}{\left(X_{m}^{2}+Z_{p}^{2}\right)^{2}}-\frac{2 z z^{\prime} Z_{p}\left(3 X_{m}^{2}-Z_{p}^{2}\right)}{\left(X_{m}^{2}+Z_{p}^{2}\right)^{3}}\right)
\end{aligned}
$$




$$
\begin{aligned}
G_{x z v}=\frac{X_{m}}{4 \pi} \frac{1-2 v^{\prime}}{1-v^{\prime}} & {\left[\left(\frac{1}{X_{m}^{2}+Z_{p}^{2}}-\frac{1}{X_{m}^{2}+Z_{m}^{2}}\right)+\frac{2}{1-2 v^{\prime}}\left(\frac{Z_{p}^{2}}{\left(X_{m}^{2}+Z_{P}^{2}\right)^{2}}-\frac{Z_{m}^{2}}{\left(X_{m}^{2}+Z_{m}^{2}\right)^{2}}\right)\right] } \\
- & \frac{X_{m}}{2 \pi} \frac{1}{1-v^{\prime}} \times\left(4\left(1-v^{\prime}\right) \frac{z Z_{p}}{\left(X_{m}^{2}+Z_{p}^{2}\right)^{2}}+\frac{2 z z^{\prime} Z_{p}\left(3 Z_{p}^{2}-X_{m}^{2}\right)}{\left(X_{m}^{2}+Z_{p}^{2}\right)^{3}}\right)
\end{aligned}
$$

\section{References}

1. Herderick, E., Additive manufacturing of metals: A review. Materials science and technology, 2011. 1413.

2. Namatollahi, M., et al., Additive manufacturing (AM), in Metals for Biomedical Devices. 2019, Elsevier. p. 331-353.

3. Camacho, D.D., et al., Applications of additive manufacturing in the construction industry-A forwardlooking review. Automation in construction, 2018. 89: p. 110-119.

4. Ngo, T.D., et al., Additive manufacturing (3D printing): A review of materials, methods, applications and challenges. Composites Part B: Engineering, 2018. 143: p. 172-196.

5. Ji, X., et al., Analytical modeling of post-printing grain size in metal additive manufacturing. Optics and Lasers in Engineering, 2020. 124: p. 105805.

6. Tabei, A., et al., Modeling of texture development in additive manufacturing of Ni-based superalloys. The International Journal of Advanced Manufacturing Technology, 2019: p. 1-10.

7. Bartlett, J.L., et al., Revealing mechanisms of residual stress development in additive manufacturing via digital image correlation. Additive Manufacturing, 2018. 22: p. 1-12.

8. Roehling, J.D., et al., Reducing residual stress by selective large-area diode surface heating during laser powder bed fusion additive manufacturing. Additive Manufacturing, 2019. 28: p. 228-235.

9. Wang, Z., et al., Residual stress mapping in Inconel 625 fabricated through additive manufacturing: Method for neutron diffraction measurements to validate thermomechanical model predictions. Materials \& Design, 2017. 113: p. 169-177.

10. An, K., et al., Neutron residual stress measurement and numerical modeling in a curved thin-walled structure by laser powder bed fusion additive manufacturing. Materials \& design, 2017. 135: p. 122-132.

11. Denlinger, E.R., J.C. Heigel, and P. Michaleris, Residual stress and distortion modeling of electron beam direct manufacturing Ti-6Al-4V. Proceedings of the Institution of Mechanical Engineers, Part B: Journal of Engineering Manufacture, 2015. 229(10): p. 1803-1813.

12. Zhao, X., et al., Numerical modeling of the thermal behavior and residual stress in the direct metal laser sintering process of titanium alloy products. Additive Manufacturing, 2017. 14: p. 126-136.

13. Romano, S., et al., Fatigue properties of AlSi10Mg obtained by additive manufacturing: Defect-based modelling and prediction of fatigue strength. Engineering Fracture Mechanics, 2018. 187: p. 165-189.

14. Noronha, P. and J. Wert, An ultrasonic technique for the measurement of residual stress. Journal of Testing and Evaluation, 1975. 3(2): p. 147-152.

15. Chung, D., Thermal analysis of carbon fiber polymer-matrix composites by electrical resistance measurement. Thermochimica acta, 2000. 364(1-2): p. 121-132.

16. Krause, T.W., et al., Investigation of the stress-dependent magnetic easy axis in steel using magnetic Barkhausen noise. Journal of Applied Physics, 1996. 79(8): p. 4242-4252.

17. Ager III, J.W. and M.D. Drory, Quantitative measurement of residual biaxial stress by Raman spectroscopy in diamond grown on a Ti alloy by chemical vapor deposition. Physical Review B, 1993. 48(4): p. 2601.

18. Prime, M.B., Cross-sectional mapping of residual stresses by measuring the surface contour after a cut. Journal of engineering materials and technology, 2001. 123(2): p. 162-168.

19. Aggarangsi, P. and J.L. Beuth. Localized preheating approaches for reducing residual stress in additive manufacturing. in Proc. SFF Symp., Austin. 2006.

20. Panda, B.K. and S. Sahoo. Numerical simulation of residual stress in laser based additive manufacturing process. in IOP Conference Series: Materials Science and Engineering. 2018. IOP Publishing.

21. Chen, Q., et al., An inherent strain based multiscale modeling framework for simulating part-scale residual deformation for direct metal laser sintering. Additive Manufacturing, 2019. 28: p. 406-418.

22. Ganeriwala, R., et al., Evaluation of a thermomechanical model for prediction of residual stress during laser powder bed fusion of Ti-6Al-4V. Additive Manufacturing, 2019. 27: p. 489-502.

23. Ding, H. and Y.C. Shin, A metallo-thermomechanically coupled analysis of orthogonal cutting of AISI 1045 steel. Journal of Manufacturing Science and Engineering, 2012. 134(5): p. 051014.

24. Mirkoohi, E., et al., Heat Source Modeling in Selective Laser Melting. Materials, 2019. 12(13): p. 2052. 
25. Mirkoohi, E., et al., Thermal modeling of temperature distribution in metal additive manufacturing considering effects of build layers, latent heat, and temperature-sensitivity of material properties. Journal of Manufacturing and Materials Processing, 2018. 2(3): p. 63.

26. Fergani, O., et al., Analytical modelling of residual stress in additive manufacturing. Fatigue \& Fracture of Engineering Materials \& Structures, 2017. 40(6): p. 971-978.

27. Carslaw, H.S. and J.C. Jaeger, Conduction of heat in solids. Oxford: Clarendon Press, 1959, 2nd ed., 1959.

28. Saif, M., C. Hui, and A. Zehnder, Interface shear stresses induced by non-uniform heating of a film on a substrate. Thin Solid Films, 1993. 224(2): p. 159-167.

29. Cowper, G., The shear coefficient in Timoshenko's beam theory. Journal of applied mechanics, 1966. 33(2): p. 335-340.

30. McDowell, D., An approximate algorithm for elastic-plastic two-dimensional rolling/sliding contact. Wear, 1997. 211(2): p. 237-246.

31. Qi, Z., B. Li, and L. Xiong, An improved algorithm for McDowell's analytical model of residual stress. Frontiers of Mechanical Engineering, 2014. 9(2): p. 150-155.

32. Khan, A.S. and S. Huang, Continuum theory of plasticity. 1995: John Wiley \& Sons.

33. Lesuer, D., Experimental investigation of material models for Ti-6Al-4V and 2024-T3. Avaliable online: https://e-reports-ext. llnl. gov/pdf/236167. pdf (accessed on 29 December 2015), 2000.

34. Group, A.M., J. Merwin, and K. Johnson, An analysis of plastic deformation in rolling contact. Proceedings of the Institution of Mechanical Engineers, 1963. 177(1): p. 676-690.

35. Welsch, G., R. Boyer, and E. Collings, Materials properties handbook: titanium alloys. 1993: ASM international.

36. Heigel, J., P. Michaleris, and E.W. Reutzel, Thermo-mechanical model development and validation of directed energy deposition additive manufacturing of Ti-6Al-4V. Additive manufacturing, 2015. 5: p. 919.

37. amshidinia, M., F. Kong, and R. Kovacevic, Numerical modeling of heat distribution in the electron beam melting® of Ti-6Al-4V. Journal of Manufacturing Science and Engineering, 2013. 135(6): p. 061010.

38. Li, J.J., W.L. Johnson, and W.-K. Rhim, Thermal expansion of liquid Ti-6Al-4V measured by electrostatic levitation. Applied physics letters, 2006. 89(11): p. 111913.

39. Mills, K.C., Recommended values of thermophysical properties for selected commercial alloys. 2002: Woodhead Publishing.

40. Murgau, C.C., Microstructure model for Ti-6Al-4V used in simulation of additive manufacturing. 2016, Doctoral thesis, Luleå University of Technology.

41. Nadammal, N., et al., Influence of support configurations on the characteristics of selective laser-melted inconel 718. JOM, 2018. 70(3): p. 343-348.

42. Prevey, P.S., X-ray diffraction residual stress techniques. ASM International, ASM Handbook., 1986. 10: p. 380-392.

43. Selvan, J.S., et al., Laser boronising of Ti-6Al-4V as a result of laser alloying with pre-placed BN. Materials Science and Engineering: A, 1999. 260(1-2): p. 178-187. 